\section{Towards a better control over science}

SIR-The desire to understand the world has today been overtaken by the necessity of exploiting it. Scientific results and advances are for the most part produced within institutions whose ultimate aims are technological. The direction in which research, whether 'fundamental' or 'applied', proceeds is governed by economic, social, health-related or military considerations.

This orientation cannot be ignored by research workers and society has the right to pass judgement on it. With its reductionist viewpoint and disregard for all other forms of truth and knowledge, science puts itself in conflict with nature, culture and individuals.

Thus, unless science can be brought under control, it represents a serious risk to the environment, to mankind and to individuals. Scientific development continues, however, and promotes its own acceleration, with the naive assent of a society whose only vision of the future is expressed in terms of technological artefacts, although the identification of scientific achievement with progress, or even happiness, is largely imaginary. The increasing rate of scientific output is causing a qualitative change in the dependence of the individual on science. This is obvious for the practical aspects of life, which are constantly being altered by technology, but also holds for its more private aspects. The concepts of subjectivity, privacy and secrecy are being vigorously attacked by scientific disciplines which probe deeper and deeper and, while they are unable to understand everything, claim to explain everything. In the name of scientific truth, life is reduced to its measurable aspects. The increasingly narrow specialization of scientists encourages their shortsightedness as to their role in society and creates insurmountable barriers between the different scientific disciplines.

It is certainly difficult to turn back from technological advances resulting from scientific endeavour, which themselves create new demands in an industrial spiral which neither scientists nor consumers can control. We believe that clear thinking is more important than efficiency and the direction of research more important than the speed at which it is accomplished. We believe that reflection should precede any scientific project rather than following any innovation made. We believe this reflection has to be philosophical rather than technical in nature, and that it should take place across interdisciplinary barriers and include the whole of society.

J. Arsac (Informatique, Univ. Paris VI); M. BOUNias (Biochimie, Univ. Avignon); $M$ CAsse (Astrophysique, CEA Saclay); J-P.
Deleage (Physique, Univ. Paris VII); A. GSPONER (Physique des hautes énergies, ISRI, Genève); A. Jacouard (Génétique, INED, Paris); J-M. Levy-Leblond (Physique Théorique, Univ. Nice); J-M. MEYER (Embryologie, Univ. Strasbourg); F. B. Michet (Pneumologie, Univ. Montpellier); J. PANIJEL (Immunologie, CNRS-Pasteur); B. Prum (Statistique médicale, Univ. Paris V) J-P. RENARD (Embryologie, INRA-Pasteur) J-C. SAlomon (Cancérologie, CNRS, Villejuif); J-P. SCHEIDECKer (Astronomie, CNRS, Nice) J-P. Shapira (Physique nucléaire, Orsay); M. SinTzoff (Informatique, Univ. Louvain); J. TestarT* (Biologie, INSERM, Clamart).

*Corresponding author: J. Testart, INSERM U. 187, Hôpital Antoine Béclère (92141) CLAMART, France.

\section{Ozone depletion}

SIR-Some of the views in your recent leading article on ozone cannot be allowed to pass unchallenged. Those working in the field will smile wryly at the thought that financial support has been generous. Some satellite instruments were designed under cost constraints that precluded planning for extended operation. Long-term monitoring activities have been despised as 'routine', and support for them severely cut back. Problems vary from country to country. In the United States, it seems easier to get support for building instruments than for providing the resources to make prompt and good use of the data acquired. In Britain, lack of capital (and over-rigid accounting rules) has severely limited the capacity to build or deploy suitable instruments. World-wide, many major field and laboratory studies could not have been undertaken without substantial support from the Chemical Manufacturers' Association.

Insufficient funds also explain why the arguments used in discussing the environmental effects of ozone depletion are so often emotive. Recommendations have been made at intervals since 1974 for comprehensive long-term research into the effects on man, animals, plants and materials of increased fluxes of ultraviolet radiation. They have yet to be acted upon.

The most pressing question, however, is this - is current understanding of the processes that control the abundance of ozone sufficient to justify existing policies on halocarbon emissions? There is no longer any doubt that halocarbons are primarily responsible for the effects observed in Antarctica. The Ozone Trends Panel has found definite evidence outside Antarctica for ozone depletion over two decades, overlain by effects of the solar cycle. The observed cyclic change of ozone is simulated fairly well by models. However, when allowance is made for increase of tropospheric ozone ${ }^{2}$, these models significantly underestimate the underlying trend in stratospheric ozone. There are no grounds for compla- cency - the assumption in the Montreal Protocol that the halogen content of the atmosphere can be allowed to increase is no longer tenable. The protocol must be swiftly ratified, and strengthened.

J.C. FARMAN

British Antarctic Survey,

High Cross,

Madingley Road,

Cambridge CB3 OET, UK

Nanure 332. 291 (1988)

Penkett, S. A. Nature 332, 204-205 (1988)

\section{Stations in space}

SIR-The anonymous writer of your editorial "Stations in space" (Nature 332, $292 ; 1988)$ seems not to have thought very deeply about the future of space exploration. The most important 'purpose' of the space station is the experience that will be gained during its construction. All other suggested purposes are, to some extent, icing on the cake. The future of space exploration, including that of space science carried out from Earth orbit, will largely depend on the ability to manufacture large structures in space, and the building of the space station will pioneer the necessary technology.

It should be self-evident that the continued manned (or even large-scale unmanned) exploration of the Solar System will depend on the assembly of large spacecraft in Earth orbit. The experience to be gained by the construction of the space station will be an essential prerequisite to these ventures. Moreover, while your writer is quite right about the immense value to astronomy of the space telescope, this instrument should not be seen as the culmination of space astronomy. It is certainly possible, in principle, to build larger instruments, but, again, these will have to be assembled in space and the above argument is just as valid. Important though the space telescope is in the short term, history is likely to show that the ability to construct large space structures (telescopes, space ships and so on) will be of more lasting scientific value. Make no mistake, this technology, carried to its logical conclusion, will enable us to send instruments to the stars.

Finally, large-scale investment in space is important because it provides business for high-technology companies which would otherwise be producing weapons. This is important because only when such alternative business is found will disarmament become acceptable to the US military-industrial complex. In fact, given the magnitude of US military spending, the argument that the federal deficit makes the space station an irresponsible undertaking looks a little silly.

University of London Observatory,

AN CRAWFORD

Mill Hill Park,

London NW7 2OS, UK 